\title{
Peripheral Facial Paresis and COVID-19 Case Report
}

\author{
Marius Militaru ${ }^{1,2, *}$, Daniel Florin Lighezan ${ }^{3,4}$, Manuela Narcisa Petrescu ${ }^{3}$, and Anda Gabriela Militaru ${ }^{3,4}$ \\ ${ }^{1}$ Department of Neuroscience, Discipline of Neurology, "Victor Babes" University of Medicine and Pharmacy Timisoara, Timisoara, Romania \\ ${ }^{2}$ Neurology, Municipal Emergency Hospital Timisoara, Timisoara, Romania \\ ${ }^{3}$ Department of Internal Medicine I, Discipline of Medical Semiology I, "Victor Babes" University of Medicine and Pharmacy Timisoara, Timisoara, \\ Romania \\ ${ }^{4}$ Internal Medicine, Municipal Emergency Hospital Timisoara, Timisoara, Romania
}

*Corresponding author: Marius Militaru, Department of Neuroscience, Discipline of Neurology, "Victor Babes" University of Medicine and Pharmacy Timisoara, Eftimie Murgu Sq. no. 2, 300041, Timisoara, Romania, E-mail: marius.militaru@umft.ro

Received: 02 Jun, 2021 | Accepted: 12 Jul, 2021 | Published: 20 Jul, 2021

Citation: Militaru M, Lighezan DF, Petrescu MN, Militaru AG (2021) Peripheral Facial Paresis and COVID-19 Case Report. J Neurol Neurobiol 7(1): dx.doi.org/10.16966/2379-7150.176

Copyright: (c) 2021 Militaru M, et al. This is an open-access article distributed under the terms of the Creative Commons Attribution License, which permits unrestricted use, distribution, and reproduction in any medium, provided the original author and source are credited.

\section{Abstract}

Among the neurological complications that occurred during Corona Virus Disease 2019 (COVID-19) infection, peripheral facial paresis is one of the conditions that can occur both during and after the Severe Acute Respiratory Tract Syndrome Corona Virus 2 (SARS-CoV-2) infection period. We present the case of a young man, aged 29 years, who presented the appearance of a peripheral facial paresis on the $14^{\text {th }}$ day after the onset of symptoms, isolation and PCR detection of SARS-CoV-2 infection. The patient was evaluated clinically, neurologically, underwent laboratory tests, EKG, chest Radiography, Magnetic Resonance Imaging (MRI)/Magnetic resonance Angiography (MRA) and received corticotherapy, gastric antisecretory medication, antiviral medication, vitamin B group, neurotrophic medication and facial gymnastics, with good evolution and complete remission of clinical symptoms after 21 days. It is particularly important to carefully evaluate patients during the isolation period, symptomatic/asymptomatic for COVID-19 infection, as a possible complication could be peripheral facial paresis, which is why, we should evaluate, detect and treat early to reduce any persistent long-term neurological effects. Future studies are needed to identify and correlate peripheral facial paresis with SARS-CoV- 2 infection as well as to detect the causes of neurological complications during or after COVID-19 infection.

Keywords: Peripheral facial paresis; Neurological complications; COVID-19; Facial nerve

\section{Introduction}

Corona virus disease 2019 (COVID-19) is caused by severe acute respiratory tract syndrome corona virus 2 (SARS-CoV-2), and respiratory symptoms are the main clinical features [1,2]. SARS-CoV-2 is an RNA virus, which has a strong human-to-human transmission impact thus contributing to a high outbreak of COVID-19 [3]. This virus is extremely infectious and has caused an outbreak of pneumonia which spread from Wuhan, China around the world [1-4].

The ongoing COVID-19 pandemic has affected millions of people worldwide and although COVID-19 mainly causes respiratory symptoms in humans, some patients may also have gastrointestinal, neurological, cardiovascular and other symptoms [1,2]. Although the neurological complications of COVID-19 are rarely described, recent work has shown damage to the nervous system, with several neurological symptoms and complications related to this infection including headache, dizziness, encephalopathy, anosmia, ageusia, encephalitis, Guillain-Barré syndrome, myelitis as well as cerebrovascular complications, which can occur in a significant proportion of patients [4-6].

Bell's palsy is an acute facial nerve condition known to be the most common cause of peripheral facial paralysis and accounts for $60 \%$ to
$70 \%$ of all causes of unilateral facial paralysis [7]. The first symptoms may be pain in the mastoid region and may cause facial paralysis [7]. Acute paresis of the facial nerve occurs frequently in clinical practice and is associated with considerable suffering due to possible functional and aesthetic sequelae [7]. The etiology and pathological mechanism are still controversial [8]. Although there is no direct evidence, more and more indirect evidence suggests that there are many potential mechanisms involved in the occurrence of Bell's palsy, some these being closely associated with viral infections, and having the characteristic of seasonal prevalence $[8,9]$.

The main predispositions of Bell's palsy include cold, fatigue, low endurance and poor immunity [9]. Bell's palsy usually occurs 1-2 weeks after viral infection, which could be autoimmune demyelinating disease following virus infection, with most studies suggesting that Bell's palsy is linked to an immune response after viral infection $[9,10]$. The genome of the Herpes Simplex Virus 1 (HSV-1) found in the endoneurial fluid of the facial nerve of patients with Bell's palsy has supported the hypothesis of HSV-1 reactivation in the geniculate ganglion and inflammation in the labyrinthine segment, which is thought to be the pathogenic mechanism of Bell's palsy [11].

In 2020, two articles were published in which an asymptomatic case of COVID-19 pneumonia complicated by acute cerebral infarction and 
another case of COVID-19 pneumonia complicated by encephalitis were, respectively, reported $[3,12]$. These cases have suggested that COVID-19 may develop, neurological symptoms, initially or during the infection, especially in patients with asymptomatic or mild infection [3,12]. In this case we report a patient diagnosed with moderate symptoms of COVID-19 complicated by Bell's palsy started on the $14^{\text {th }}$ day after the onset of SARS-CoV-2 infection.

\section{Case Report}

We present the case of a young man, 29-years-old, non-smoker, without cardiovascular risk factors present, without associated vascular, autoimmune or oncological pathology, who on November 17,2020 has clinical symptoms specific to SARS-CoV-2 infection. The Real-Time Reverse Transcription-Polymerase Chain Reaction (RTPCR) results for SARS-CoV-2 RNA in nasal and oropharyngeal swabs were positive fact which confirms positivity to COVID-19 infection and isolates at home. The patient initially showed an accentuated state of fatigue, followed by the second day of infection of muscular pains, especially at the level of the upper limbs, and from the third day he presented the loss of smell and taste. In association, he presented a mild state of anxiety caused by the patient's report of SARSCoV-2 infection and the uncertainty of the evolution of his health during that period. The patient did not show symptoms related to shortness of breath, cough, chest pain or fever. The patient did not show vertigo during SARS-CoV-2 infection, right temporoparietal headache appearing on day 14 from COVID-19 infections. The patient's assessment did not detect the presence of existing malignancy, weight loss or recent infections. The patient was not exposed to toxic substances or sudden weather changes in the period prior to SARS-CoV-2 symptoms.

Throughout the isolation period at home for COVID-19 infection, the patient was monitored and evaluated by telephone by a general practitioner with a favorable outcome. Muscle weakness and pain persisted in the first 10 days of infection, and the smell reappeared on the $12^{\text {th }}$ day after infection, the taste reappeared about 1 month after the onset of COVID-19 infection.

The patient's Blood Pressure (BP) was maintained as follows: Systolic Blood Pressure (SBP) in the range of $120-130 \mathrm{mmHg}$ and Diastolic Blood Pressure (DBP) in the range of 70-80 mmHg. Heart Rate (HR) was maintained in the range of $60-80 \mathrm{~b} /$ minute, and oxygen saturation $\left(\mathrm{SaO}_{2}\right)$ in the range of $95-96 \%$. The temperature was in the range of 36.2-36.8 Celsius degrees. The patient received treatment with vitamin $\mathrm{C}$ and $\mathrm{D}$, gastric antisecretory, with favorable evolution, in 13 days from the first symptoms of COVID-19 showed no symptoms of severe SARS-CoV-2 infection symptoms.

On November 30,2020 , on the $14^{\text {th }}$ day after the onset of clinical symptoms and PCR detection of SARS-CoV-2 infection, the patient presented with right lagophthalmia, right facial asymmetry and right temporo-parietal headache, transient paresthesias in the right hemiface, without signs of herpes zoster presence, which is why on December 1,2020 , on the $15^{\text {th }}$ day after the onset of clinical symptoms of COVID-19 he presented to the emergency department.

At the clinical examination, the patient was without fever, his temperature measuring 36.2 Celsius degrees; he was well oriented in time and space, without presenting pathological elements in the evaluation on cardiovascular, pulmonary or gastrointestinal systems, without presence of edema, without existing lymphadenopathy. The SBP was $125 \mathrm{mmHg}$, DBP was $55 \mathrm{mmHg}$, HR $78 \mathrm{~b} /$ minute and 95\% $\mathrm{SaO}_{2}$.
An electrocardiogram (ECG) is performed that shows a sinus rhythm, HR $80 \mathrm{~b} /$ minute, no pathological elements are detected.

Chest radiography does not show pathological elements, a normal heart and aorta, without pulmonary condensation processes or radiographic pleural fluid collections.

The neurological evaluation showed a conscious, cooperative patient, spatially and temporally oriented, without signs of meningeal irritation, possible gait, Romberg negative, without highlighting elements of motor deficiency of the upper or lower limbs in comparative tests, osteotendinous reflexes present, plantar skin reflex in bilateral plantar flexion present, eyes with parallel axes, equally reactive pupils. Elements of peripheral facial paresis have been highlighted. Facial palsy was graded according to the House-Brackmann scale [13], moderate dysfunction grade 3 (right eye lagophthalmia, wiping of facial and nasolabial folds on the right side of the face and right facial asymmetry, the commissure of the mouth being right side), the other cranial nerves evaluated being without pathological elements. The patient cannot wrinkle his forehead on the right side, cannot blink on the right side, cannot pronounce the letters $\mathrm{B}, \mathrm{M}, \mathrm{P}$, chewing is influenced by the accumulation of food on the affected side. The corneal reflex is abolished on the right side. The patient did not present hyperacusis (hypersensitive to sound), ipsilateral reduced lacrimal fluid production, or reduced salivation. The patient presented loss of taste not only on ipsilateral $2 / 3$ of the tongue; he presented loss of smell and taste on the third day of COVID-19 infection.

The laboratory tests performed show a slight increase in leukocytes value and increased granulocytes as well as a slight decrease of lymphocytes and monocytes, without other changes in the blood count or biochemical parameters. The list of all tests performed in the emergency service is reported in table 1 .

At 2 days after the detection of the symptoms of peripheral facial paresis, 25-hydroxy-vitamin D (25-OH-vitamin-D) is measured, which shows a low level of $23 \mu \mathrm{g} / \mathrm{L}(59.7 \mathrm{nmol} / \mathrm{L})$ and he received treatment with vitamin $\mathrm{D}$. Electromyographic and nerve conduction study was not performed. On December 3, 2020, due to the persistence of right temporo-parietal headache, in addition to the symptoms of peripheral facial paresis and lack of smell, it was decided to perform a Contrast-Enhanced (CE) MRI and MRA that shows normal intracerebral and extracerebral fluid spaces. Normal looking intracerebral and extracerebral brain structures, without hypersignal of the facial nerve in the geniculate ganglion region corresponding to its inflammation/oedema. The cisternal, intracanalicular, tympanic, mastoid and geniculate ganglion segments of the facial nerve were without signal modifications, without restriction of diffusion, without pathological contrast. Pituitary gland and orbits of normal aspect. No pathological CE outlets. Normal appearance of facial sinuses and mastoid cells. Right side nasal septum deviation. MRA: without pathological changes in the cerebral arteries of the Willis polygon and the vertebra basilar system.

At 5 days from the onset of peripheral facial paresis, the patient associates a slight chest pain, which is why a pneumological consultation is performed that does not show pathological changes.

The patient initially undergoes, in the emergency room, corticosteroid treatment with methylprednisolone $125 \mathrm{mg}$ in IV infusion and famotidine $20 \mathrm{mg}$ in IV infusion. Oral corticosteroid therapy is instituted with prednisone $5 \mathrm{mg}, 1 \mathrm{mg} / \mathrm{kg}$ body weight, 60 $\mathrm{mg} /$ day ( $30 \mathrm{mg} /$ day in the morning, $30 \mathrm{mg} /$ day at lunch) with a decrease of $5 \mathrm{mg}$ every 2-3 days over a period of 21 days and monitoring of the clinical evolution. He receives treatment with gastric antisecretory 
Table 1: Highlighting the biological parameters performed in the case of the patient with peripheral facial paresis and SARS-CoV-2 infection.

\begin{tabular}{|l|c|c|}
\hline \multicolumn{1}{|c|}{ Biological parameters } & Values & Reference interval \\
\hline Leucocites $\left(10^{3} / \mu \mathrm{L}\right)$ & 9.8 & $4.0-9.5$ \\
\hline Granulocytes $(\%)$ & 83.4 & $45-70$ \\
\hline Lymfocites $(\%)$ & 14.4 & $20-40$ \\
\hline Monocytes $(\%)$ & 2.2 & $3.9-9.5$ \\
\hline Granulocytes $\left(10^{3} / \mu \mathrm{L}\right)$ & 8.2 & $1.8-6.7$ \\
\hline Lymfocites $\left(10^{3} / \mu \mathrm{L}\right)$ & 1.4 & $0.8-3.8$ \\
\hline Monocytes $\left(10^{3} / \mu \mathrm{L}\right)$ & 0.2 & $0.1-0.9$ \\
\hline Erithrocytes $\left(10^{6} / \mu \mathrm{L}\right)$ & 5.09 & $4.5-5.9$ \\
\hline Hemoglobin $(\mathrm{g} / \mathrm{dl})$ & 14.6 & $13.6-17.2$ \\
\hline Hematocrit $(\%)$ & 44.6 & $(39-51)$ \\
\hline Thrombocytes $\left(10^{3} / \mu \mathrm{L}\right)$ & 281 & $150-400$ \\
\hline ALT (U/L) & 29 & $0-50$ \\
\hline AST (U/L) & 24 & $17-59$ \\
\hline CK (U/L) & 84 & $30-170$ \\
\hline CKMB (U/L) & 11 & $0-16$ \\
\hline Creatinine (mg/dL) & 1 & $0.8-1.5$ \\
\hline Glycemie $(\mathrm{mg} / \mathrm{dL})$ & 104 & $65-105$ \\
\hline LDH (mg/dL) & 173 & $120-246$ \\
\hline Urea (mg/dL) & 40 & $19-43$ \\
\hline K (mmol/l) & 4.5 & $3.5-5$ \\
\hline Na (mmol/l) & 138 & $135-145$ \\
\hline
\end{tabular}

AAT: Alanine Aminotransferase; AST: Aspartate Aminotransferase; CK: Creatine Kinase; CKMB: Creatine Kinase MB; LDH: Lactate Dehydrogenase; K: Potassium; Na: Sodium

medication, omeprazole $20 \mathrm{mg} / \mathrm{day}$, throughout the corticosteroid treatment without gastric complaints, vitamin B1, B6, B12 and vitamin $\mathrm{D}$ treatment for a period of 21 days and antiviral medication aciclovir $400 \mathrm{mg}, 1200 \mathrm{mg} / \mathrm{day}$, for 7 days. In order to avoid dryness of the cornea, artificial tears treatment is instituted throughout the maintenance of the open eye due to peripheral facial paresis and the use of an eye bandage to keep the eye closed, especially during sleep. The patient is recommended to adopt a salt-free, hypoglycemic diet and to periodically control his blood pressure to avoid possible side effects caused by corticosteroid treatment. The patient follows medical recovery treatment by facial gymnastics every day consisting of exercises to improve the coordination of the facial muscles, exercises for facial movements for functions as speaking or closing the eyes, exercises for facial expressions, for a period of 21 days.

The patient's evolution is favorable throughout the treatment, without complications or side effects of medication, with decreased symptoms of peripheral facial paresis from day 7 of drug treatment and gymnastics and complete remission of clinical symptoms after 21 days, at the end treatment the patient presenting facial symmetry, without erasing the straight facial grooves, complete closing of the right eye, without paresthesia at the level of the right hemiface.

The patient no longer has a headache, remission of chest pain, and the taste reappeared about a month after the onset of peripheral facial paresis. With the remission of COVID-19 symptoms and peripheral facial paresis, the patient's state of anxiety was remitted.

\section{Discussion}

The most common cause of peripheral facial paresis is idiopathic [14]. COVID-19 is an extremely infectious disease, which mainly causes respiratory symptoms. However, there are patients who may also have neurological symptoms. We report a case of COVID-19 infection complicated with Bell's palsy with onset on the $14^{\text {th }}$ day after the onset of infection and with complete remission of symptoms after 21 days of treatment. This case suggests that Bell's palsy may be associated with COVID-19 and this infection may be a potential cause of facial paralysis. From a pathophysiological point of view, it has been suggested that peripheral facial paresis may result from facial nerve reactivity in HSV-1 infection and that, as such, episodes of peripheral facial paresis may be caused by stress (or trauma, for example after dental treatment) [15].

Current treatment options are represented by corticosteroid treatment which has been shown to significantly improve motor recovery [16]. According to a meta-analysis, antiviral medication may be associated with efficacy in reducing the risk of late sequelae (crocodile tears and motor synkinesis), [17] with possible effect in motor recovery in the presence of the associated HSV-1 infection. Physiotherapy could be effective in reducing sequelae in acute cases and improving long-term facial function in chronic cases [18].

In the case of our patient, the onset of Bell's palsy symptoms was 14 days after COVID-19 infection. Cases of patients with COVID-19 with Bell's palsy have been documented in several articles. There is a case published in 2020 with idiopathic facial paresis that occurred simultaneously with a major episode of anxiety secondary to COVID-19 infection and subsequent socio-economic repercussions [19]. In the case of our patient, there was a degree of anxiety caused by the presence of SARS-CoV-2 infection and the uncertainty of the evolution of the infection and the consequences of the infection but also of the possible sequelae caused by long-term peripheral facial paresis at the time of its occurrence. There is another recent study that showed a bidirectional association between anxiety and peripheral facial paresis, with a risk of peripheral facial paresis of 1.53 (95\%CI) $[1.21,1.94], \mathrm{P}<0.001$, in patients with the presence of anxiety [20]. In a study from China regarding the increase in anxiety and/or depression in the Chinese population during the COVID-19 pandemic, it was highlighted that these issues are related to three major psychosocial stressors: fear of infection for oneself or family, worries about loss of income, job, study, rent or general inconvenience or the inconvenience of quarantine at home [21].

It is important to highlight the effects of the COVID-19 pandemic and the implications of the socio-economic impact that must not be forgotten in the fight against SARS-CoV-2. Indeed, even as the pandemic subsides, its long-term socio-economic and psychological sequelae are likely to exist and lead to major increases in anxiety or to effects and clinical consequences favored by anxiety [19].

Peripheral facial paresis may occur before COVID-19 infection is detected. In 2020, a case was published in a pregnant woman with a full-term pregnancy, diagnosed with COVID-19 after the appearance of an isolated peripheral facial paresis. A 35-year-old, primiparous pregnant woman, at 39 weeks of gestation, presented urgently with a 2-day onset of left facial asymmetry and ipsilateral lagophthalmia. The patient had no history of diabetes, hypertension, being overweight or immunosuppression. She had no other symptoms, including fever, dyspnea, odynophagia, swelling of the face, cough, rash, myalgia, anosmia, ageusia or diplopia. She had no history of tick bites or recent trauma. She had no recent history in the epidemiological context of 
respiratory infection or SARS-CoV-2. She tested positive by nasal PCR for SARS-CoV-2 infection. At the neurological examination she was diagnosed with left peripheral facial paresis and received $60 \mathrm{mg} /$ day corticosteroid treatment with progressive dose reduction and favorable physiotherapy and remission of neurological symptoms after 15 days. She gave birth naturally to a healthy fetus with an Apgar score of 5 out of 10 with a favorable evolution, which was tested negative for SARS-CoV-2 infection [22].

Signs and neurological complications should be evaluated in all patients with SARS-CoV-2 infection. In a study published in 2020, neurological symptoms were reported in $36.5 \%$ of 19 positive COVID patients [23]. In another study, peripheral nervous system damage was reported in $8.9 \%$ of COVID-19 cases [24]. The occurrence of acute demyelinating neuropathy secondary to SARS-CoV-2 mediated immunity, Guillain-Barré syndrome, has also been reported [25].

SARS-CoV-2 is very similar in structure and infectious mechanism to other known Corona Viruses (CoV) [22]. Because neuroinvasive inclination is a common feature of $\mathrm{CoV}$, it is very likely that SARSCoV-2 also has potential neurotropism, possibly through direct neurological damage, because it has a high affinity for Angiotensin Converting Enzyme 2 (ACE2) receptors, which are expressed in the nervous system [26]. The ACE2 receptor is highly expressed in the nasal mucosa, especially in the ciliated epithelium and goblet cells where there appears to be the highest viral replication, as evidenced by the highest viral titers evaluated in the nose. SARS-CoV-2 affects the olfactory nerve and bulb that provides a direct pathway to the central nervous system [27]. It is found that ACE2 can be bound to corona virus and trigger functional changes in the ACE2/Angiotensin II Type 2 Receptor (AT2R), resulting in an imbalance in the steady state axis of cytokine regulation and a storm cytokine [28]

The prevalence of peripheral facial paresis is increased in pregnant women, especially in the third trimester of pregnancy and in the first week after birth possibly due to physiological changes during pregnancy such as immunosuppression, hypercoagulabilty conditions, or hormonal changes that may increase this susceptibility [29]. In addition, the functional prognosis of peripheral facial paresis appears to be worse in pregnant women, as the estimated recovery is $52 \%$ compared to $77 \%-88 \%$ in the general population [30].

Because our patient had no respiratory symptoms and considering the short-term nature of the course of treatment, it was decided to administer corticosteroid treatment, maintaining a careful observation for possible respiratory symptoms. Therefore, the importance of early management of corticosteroids is very important, because in the case of the presence of respiratory symptoms in COVID-19, this treatment could possibly aggravate the respiratory tract [22].

In the case of our patient, other causes of acquired facial paralysis were considered. Regarding other infectious etiologies, the absence of skin lesions and ear lesions or pain made the diagnosis of shingles chickenpox unlikely as herpes simplex virus infections. Lyme disease was also considered unlikely due to its low local prevalence, associated with a lack of tick bites and history of rashes. HIV is a major global cause of facial paralysis, but it has been ruled out. Immunological diseases such as vasculitis, sarcoidosis or other autoimmune diseases were unlikely as systemic involvement lacked any history or clinical signs. Neoplasm and cerebrovascular disease should be considered in the presence of other neurological deficits, but this was not the case. In another study, the clinical signs and laboratory characteristics of eight patients with COVID-19 who developed peripheral facial paralysis during the clinical course of the infection or as its first symptom were evaluated. In three patients, facial paralysis was the first symptom of COVID-19, while in the other five cases, it occurred 2 to 10 days after other clinical manifestations. All patients had mild and systemic respiratory symptoms of COVID-19 and none required hospitalization. According to the House Brackmann Classification System, resulting in nerve damage mild dysfunction (grade 2) in five patients and moderate dysfunction (grade 3) in three patients. Six out of seven patients were treated with oral steroids (prednisone 40-60 mg/day for 5-7 days) and one received intravenous methylprednisolone. A patient with mild manifestations received only supportive care (eye lubricant) with full recovery 2 days later. Two patients received oral acyclovir concomitantly with steroids due to a possible infection with Herpes simplex virus. Complete recovery occurred in five patients, while the other three still had some degree of facial weakness treatment at the last monitoring, 30 days after the onset of neurological symptoms [31].

In another study, the presence of peripheral facial paresis appeared from the first day of COVID 19 without herpes zoster. Although Bell's palsy was thought to be related to the virus infection, the detection of viral antigens found no evidence of other viral infections, including shingles. Surprisingly, the patient was found to be infected with SARSCoV-2. ACE2 exists in the nervous system and skeletal muscle. ACE2 expression and distribution suggest that SARS-CoV-2 may cause some neurological symptoms by direct or indirect mechanism. Therefore, it was assumed that SARS-CoV-2 infection could be associated with Bell's palsy in this reported patient. [28].

With an increasing number of COVID-19 patients worldwide, neuromuscular complications and rehabilitation concerns are becoming increasingly evident [32]. An article published in 2020 attempted to conduct a review and provide a comprehensive first summary of current knowledge of COVID-19 and peripheral nerve damage. Peripheral nerve injury may occur in patients with secondary post-infectious COVID-19; Predisposition related stretching/ compression lesions, inflammatory neuropathy, hematoma nerve attachment, or systemic neuropathies. Peripheral nerve imaging in COVID-19 patients can help characterize nerve pathology, identify the location and severity of nerve damage, and potentially elucidate injury mechanisms thus helping medical diagnosis and decisionmaking [32]. In addition, Chen C, et al. [3] reported an asymptomatic case of COVID-19 pneumonia complicated by acute stroke on March 2, 2020. Subsequently, on March 4, 2020, researchers at Beijing Ditan Hospital reported the world's first COVID-19 pneumonia complicated by encephalitis and confirmed the presence of SARS-CoV-2 RNA in the patient's cerebrospinal fluid by gene sequencing [12]. These cases suggested that COVID-19 could develop neurological symptoms, initially or during the infection, especially in patients with asymptomatic or mild infection.

Therefore, because the probability of alternative diagnosis was low and given the pandemic context, positive test for COVID-19 and the appearance of symptoms of peripheral facial paresis 14 days after infection, the presence of mild anxiety, a possible low immunity and due to a low level of vitamin $\mathrm{D}$, the presence of COVID-19 could be the potential cause of peripheral facial paresis in our patient. Thus, neurological symptoms may, in some situations, be the onset of COVID-19 infection or in other situations, such as the one presented, at an interval after the onset of COVID infection.

Damage to the facial nerve by Bell's palsy could be attributed to an immune response caused by SARS-CoV-2 both initially and during infection. This must be further confirmed by future research in terms of highlighting the signs and the initial clinical and therapeutic 
approach, during and after the SARS-CoV-2 infection of the presence of peripheral facial paresis but also of other neurological symptoms and complications.

\section{Conclusions}

Neurological symptoms, including peripheral facial paresis, may occur during (initially or during) the SARS-CoV-2 infection. However, several cases with epidemiological data are needed to support a causal relationship between the two conditions. Exploring the neurological manifestations of COVID-19 is essential for a better understanding of the virus. Therefore, further studies are needed to understand the natural history and prognostic significance of peripheral facial paresis and other neurological symptoms and complications that occurred initially, during the course of the infection or afterwards, in the SARS$\mathrm{CoV}-2$ infection and to determine the best management strategy.

\section{Author Contributions}

Conceptualization, M.M., D.F.L. and A.G.M.; methodology, M.M., A.G.M; software, M.M., M.N.P.; validation, M.M., D.F.L., and A.G.M.; formal analysis, M.M, M.N.P., D.F.L., A.G.M.; investigation, M.M, A.G.M.; resources, M.M, M.N.P., A.G.M; data creation, M.M, A.G.M.; writing, M.M, A.G.M.; original draft preparation, M.M,; writingreview and editing, M.M., A.G.M.; visualization, M.M, M.N.P., D.F.L., A.G.M; supervision, M.M, A.G.M., D.F.L. All authors have read and agreed to the published version of the manuscript.

\section{Funding}

This research received no external funding.

\section{Institutional Review Board Statement}

The study was conducted according to the guidelines of the Declaration of Helsinki, and approved by the Institutional Review Board of Medical Clinic Medicover Timisoara, Romania nr. 69424/16.03.2021.

\section{Informed Consent Statement}

Informed consent was obtained from the subject involved in the study.

\section{Conflicts of Interest}

The authors declare no conflict of interest.

\section{References}

1. Chen N, Zhou M, Dong X, Qu J, Gong F, et al. (2020) Epidemiological and clinical characteristics of 99 cases of 2019 novel corona virus pneumonia in Wuhan, China: a descriptive study. Lancet 395: 507513.

2. Guan WJ, Ni ZY, Hu Y, Liang WH, Ou CQ, et al. (2020) Clinical Characteristics of Corona virus Disease 2019 in China. N Engl J Med 382: $1708-1720$.

3. Chen C, Dong Y, Kang J, Lou S, Wan F, et al. (2020) Asymptomatic novel coronavirus pneumonia presenting as acute cerebral infarction: case report and review of the literature. Chin J Emerg Med 12: E017-E017.

4. Deng SQ, Peng HJ (2020) Characteristics of and Public Health Responses to the Corona virus Disease 2019 Outbreak in China. J Clin Med 9: 575.

5. Glavin D, Kelly D, Wood GK, McCausland BM, Ellul MA, et al. (2020) Neurological associations of COVID-19. Lancet Neurol 19: 767-783.
6. Paterson RW, Brown RL, Benjamin L, Nortley R, Wiethoff S, et al. (2020) The emerging spectrum of COVID-19 neurology: clinical, radiological and laboratory findings. Brain 143: 3104-3120.

7. Greco A, Gallo A, Fusconi M, Marinelli C, Macri GF, et al. (2012) Bell's palsy and autoimmunity. Autoimmun Rev 12: 323-328.

8. Jowett N (2018) A General Approach to Facial Palsy. Otolaryngol Clin North Am 51: 1019-1031.

9. Minnerop M, Herbst M, Fimmers R, Kaabar P, Matz B, et al. (2008) Bell's palsy: combined treatment of famciclovir and prednisone is superior to prednisone alone. J Neurol 255: 1726-1730.

10. Steiner I (1996) Human herpes viruses latent infection in the nervous system. Immunol Rev 152: 157-173.

11. McCormick DP (1972) Herpes-simplex virus as a cause of Bell's palsy. Lancet 1: 937-939.

12. Wan $Y$, Cao S, Qi Fang Q, Wang M, Huang Y (2019) Coronavirus disease 2019 complicated with Bell's palsy: a case report. Research Square 1-7.

13. House JW, Brackmann DE (1985) Facial nerve grading system. Otolaryngol Head Neck Surg 93: 146-147.

14. Heckmann JG, Urban PP, Pitz S, Guntinas-Lichius O, Gágyor I (2019) The Diagnosis and Treatment of Idiopathic Facial Paresis (Bell's Palsy). Dtsch Arztebl Int 116: 692-702.

15. Makhoul R, Even J, Sudrat Y, Devoize L, Huard C (2014) Paralysie faciale périphérique et soins dentaires: lien ou coïncidence, pour quelle prise en charge? Med Buccale Chir Buccale 20: 103-106.

16. Madhok VB, Gagyor I, Daly F, Somasundara D, Sullivan M, et al. (2016) Corticosteroids for Bell's palsy (idiopathic facial paralysis). Cochrane Database Syst Rev 18: CD001942.

17. Gagyor I, Madhok VB, Daly F, Sullivan F (2019) Antiviral treatment for Bell's palsy (idiopathic facial paralysis). Cochrane Database Syst Rev 9: CD001869.

18. Teixeira LJ, Valbuza JS, Prado GF (2011) Physical therapy for Bell's palsy (idiopathic facial paralysis). Cochrane Database Syst Rev 12: CD006283.

19. Fricain M, Moreau N (2020) Lockdown-related idiopathic facial paralysis: illustration of an indirect victim of the COVID-19 pandemic? J Oral Med Oral Surg 26: 1-4.

20. Tseng CC, Hu LY, Liu ME, Yang AC, Shen CC, et al. (2017) Bidirectional association between Bell's palsy and anxiety disorders: A nationwide population-based retrospective cohort study. J Affect Disord 215: 269-273.

21. Li J, Yang Z, Qiu H, Wang Y, Jian L, et al. (2020) Anxiety and depression among general population in China at the peak of the COVID-19 epidemic. World Psychiatry 19: 249-250.

22. Figueiredo R, Falcão V, Pinto MJ, Ramalho C (2020) Peripheral facial paralysis as presenting symptom of COVID-19 in a pregnant woman. BMJ Case Rep 13: e237146.

23. Montalvan V, Ulrich AK, Tirschwell DL, Zunt JR (2020) Neurological manifestations of COVID-19 and other coronavirus infections: A systematic review. Clin Neurol Neurosurg 194: 105921.

24. Mao L, Jin H, Wang M, Hu Y, Chen S, et al. (2020) Neurologic Manifestations of Hospitalized Patients With Coronavirus Disease 2019 in Wuhan, China. JAMA Neurol 77: 683-690.

25. Zhao H, Shen D, Zhou H, Liu J, Chen S (2020) Guillain-Barré syndrome associated with SARS-CoV-2 infection: causality or coincidence? Lancet Neurol 19: 383-384. 
26. Li YC, Bai WZ, Hashikawa T (2020) The neuroinvasive potential of SARS-CoV2 may play a role in the respiratory failure of COVID-19 patients. J Med Virol 92: 552-555.

27. Bridwell R, Long B, Gottlieb M (2020) Neurologic complications of COVID-19. Am J Emerg Med 38: 1549.

28. Ji X, Zhang C, Zhai Y, Zhang Z, Zhang C, et al. (2020) TWIRLS, an automated topic-wise inference method based on massive literature, suggests a possible mechanism via ACE2 for the pathological changes in the human host after coronavirus infection. medRxiv 20025437.

29. Hussain A, Nduka C, Moth P, Malhotra R (2017) Bell's facial nerve palsy in pregnancy: a clinical review. J Obstet Gynaecol 37: 409-415.
30. Gillman GS, Schaitkin BM, May M, Klein SR (2002) Bell's palsy in pregnancy: a study of recovery outcomes. Otolaryngol Head Neck Surg 126: 26-30.

31. Lima MA, Silva MTT, Soares CN, Coutinho R, Oliveira HS, et al. (2020) Peripheral facial nerve palsy associated with COVID-19. J Neurovirol 26: 941-944.

32. Fernandez CE, Franz CK, Ko JH, Walter JM, Koralnik IJ, et al. (2021) Imaging Review of Peripheral Nerve Injuries in Patients with COVID-19. Radiology 298: E117-E130. 